\title{
Layered Garnet Films for Applications in Atomic Traps
}

\author{
V.N. Berzhansky ${ }^{a, *}$, V.G. Vishnevskil ${ }^{b}$, H.T. Milyukova $^{b}$, A.S. Nedviga $^{b}$, \\ A.G. NesteruK ${ }^{b}$ And H.V. DANisheVskaya ${ }^{b}$ \\ V.I. Vernadsky Taurida National University, Vernadsky ave. 4, Simferopol 95007, Ukraine
}

\begin{abstract}
Layered high-coercive garnet films characterized by a couple of parameters very suitable for creating reconfigurable magnetic atomic traps with new properties are investigated. Non-uniform distribution of inner stresses and chemical composition stratification obtained in the films can be increased technologically to form sublayers for independent local switching by means of thermomagnetic recording. It makes possible to provide more than two magnetic states and create special types of atomic traps with additional "states of freedom". The experience in the films synthesis and experimental thermomagnetic recording for multilevel traps modeling is described.
\end{abstract}

PACS numbers: 37.10.Gh, 61.72.Ff, 68.55.-a, 75.50.Ss

\section{Introduction}

Monocrystalline uniaxial anisotropy high-coercive garnet films (GF) are used for thermo-magnetic recording by laser beams or contact printing. Contact printing technique is an effective tool [1] in forensic investigations. As a rule, it is expedient to decrease their Curie point $\left(T_{\mathrm{C}}<100^{\circ} \mathrm{C}\right)$. Recently [2] it has been established that the GF can be the media for formation of erasable micropotentials that assist trapping of "ultracold" neutral atoms (such as ${ }^{87} \mathrm{Rb}$ ). It was shown that some features of GF and conditions of their growth can be used to create new variants of atomic traps [3].

To increase coercivity $H_{\mathrm{c}}$ in practice it is sufficient to realize misfit strains using the positive mismatch $\Delta a=$ $a_{\mathrm{f}}-a_{\mathrm{s}}$ between crystalline lattices of film $\left(a_{\mathrm{f}}\right)$ and substrate $\left(a_{\mathrm{s}}\right)$. Induced stresses are proportional to the $\Delta a$ value, but networks of misfit dislocations are formed to relieve them. The corresponding strain-induced morphology has a great influence on films parameters. In addition to [4], both the GF morphology and their chemical composition analysis are considered below, because the space resolution of thermomagnetic recording strongly depends on them.

\section{Preparation of high-coercive garnet films}

Monocrystalline $\mathrm{Gd}_{3} \mathrm{Ga}_{5} \mathrm{O}_{12} \quad$ (GGG) substrates with (111) crystallographic orientation, only positive substrate-film lattice constants mismatch in a range of $\Delta a=0.04-0.12 \AA$ were used in the experiments of the films growth by means of liquid phase epitaxy (LPE). After mechanical polishing by diamond abrasives (up to

\footnotetext{
* corresponding author; e-mail: roton@crimea.edu
}

optical quality) and ion beam etching the thickness of GF was $h=1-2 \mu \mathrm{m}$. As a rule, this procedure slightly increases GF's coercivity $[1,4]$.

Contrary to [1] for trapping atoms it is necessary sometimes to provide $T_{\mathrm{C}} \approx 200^{\circ} \mathrm{C}$. Thus, the films mostly of $(\mathrm{Bi}, \mathrm{Sm}, \mathrm{Lu})_{3}(\mathrm{Fe}, \mathrm{Al}, \mathrm{Ga})_{5} \mathrm{O}_{12}$ composition were synthesized on GGG $\left(a_{\mathrm{s}}=12.383 \AA\right)$ using the isothermal dipping technique.

Melt compositions, defined by the parametric convention of Blank-Nielsen [5], were: $R_{1}=\mathrm{Fe}_{2} \mathrm{O}_{3} / \mathrm{Ln}_{2} \mathrm{O}_{3}=$ 6.36-7.24; $\quad R_{2}=\mathrm{Fe}_{2} \mathrm{O}_{3} / \mathrm{Me}_{2} \mathrm{O}_{3}=10-19 ; \quad R_{3}=$ $\mathrm{PbO} / \mathrm{Bi}_{2} \mathrm{O}_{3} \approx 2 ; R_{4}=\sum$ garnet $/ \sum$ melt $=0.057-0.073$.

$\mathrm{Ln}_{2} \mathrm{O}_{3}$ is the sum of rare-earth oxides, the solvent oxides are $\mathrm{PbO}-\mathrm{Bi}_{2} \mathrm{O}_{3}-\mathrm{B}_{2} \mathrm{O}_{3}$; notation $\mathrm{Me}_{2} \mathrm{O}_{3}$ means that $\mathrm{Me}$ are $\mathrm{Al}, \mathrm{Ga}$ or their combination. In the numerator position $R_{4}$ is a sum content of all garnet-forming components, in the denominator position - a sum content of all oxides including garnet-forming ones. The oxides content is presented in molar parts.

As a result, as-grown films have: coercivity $H_{\mathrm{c}}=$ 70-800 Oe, specific Faraday rotation $\theta_{\mathrm{F}}=2-2.3^{\circ} / \mu \mathrm{m}$ (wavelength of light $\lambda_{1}=532 \mathrm{~nm}$ ), saturation magnetization $4 \pi M_{\mathrm{s}} \approx 1.2 \mathrm{kG}, h=4-5 \mu \mathrm{m}$ and the Curie temperature $T_{\mathrm{C}} \approx 60-200^{\circ} \mathrm{C}$. During the experiments some samples appeared to be layered, this feature manifested itself in magnetooptic hysteresis loops [3].

\section{Magnetic and morphological properties of the films; distribution of dislocations}

Figure 1 demonstrates magnetooptic images of two types of domain structures (DS) which are obtained after demagnetizing of the same film sample's area by variable magnetic field (stable DS) and heating up to $T>T_{\mathrm{C}}$ (metastable DS). Both of them have fractal signs though maintain a hexagonal symmetry which is conditioned by 
residual cubic anisotropy in (111) plane. Fractal dimensionality of metastable DS is higher.

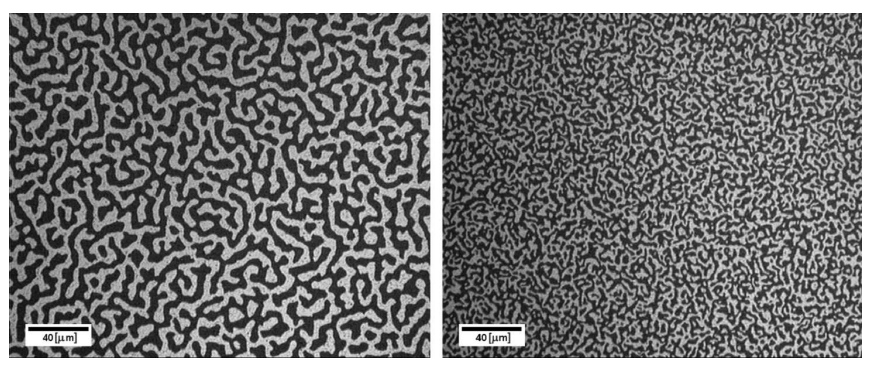

Fig. 1. Stable (left) and metastable (right) DS (polarized light).

Because the films are compressed, the "facet" surface structure is obtained. The "faceting" after the LPE is formed by intersection of dislocations with GF surface. Facet dimples are the points of emergence of dislocations half-loops which have misfit segments parallel to the interface [6]. The misfit dislocations relieve compressive stresses and the threading ones perturb remaining film's growth sufficiently to result in a "cellular"-like morphology. These "cells" and their space distribution can be observed in SEM after step-by-step ion beam etching (Fig. 2). The SEM images demonstrate the decrease of fractal dimensionality (increase of dimensions) of "cells" proportionally to the etching (exposure) time.

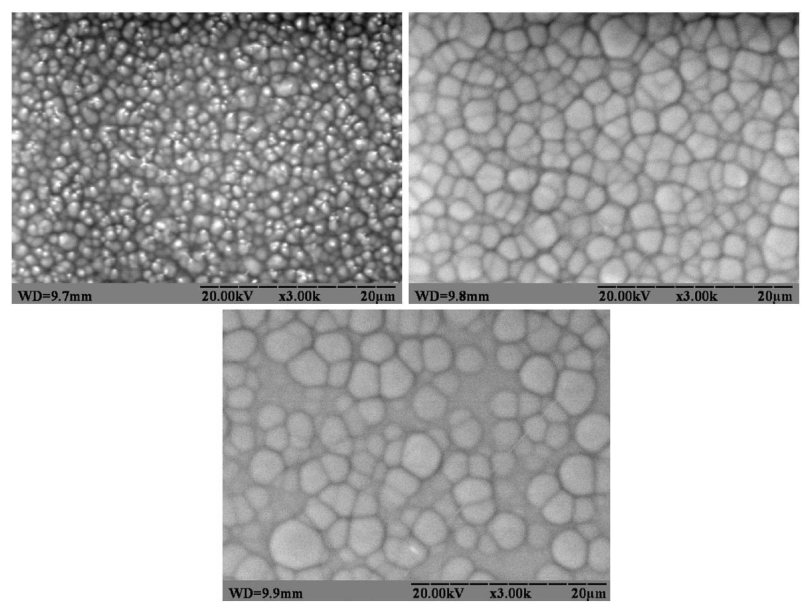

Fig. 2. "Cellular" structure of GF after step-by-step etching by the ion beam (SEM).

Figure 3 presents two SEM images of samples after selective etching: the distribution of stresses and dislocations form the non-uniform structure along the film growth direction. This uniformity explains the composition stratification in $(\mathrm{Bi}, \mathrm{Sm}, \mathrm{Lu})_{3}(\mathrm{Fe}, \mathrm{Ga}, \mathrm{Al})_{5} \mathrm{O}_{12}$ film, which was investigated with the use of energy dispersive $\mathrm{X}$-ray microanalysis system (EDS) as an equipment of SEM.
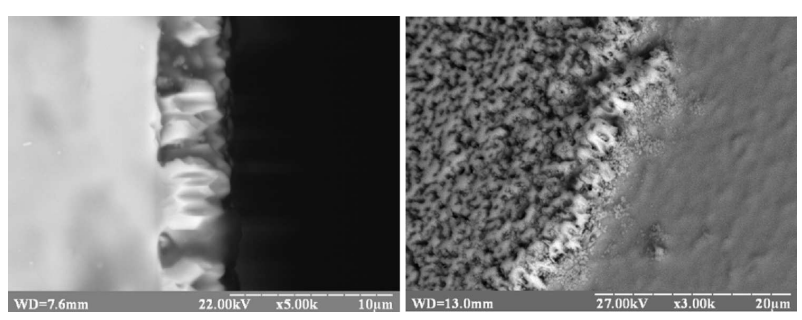

Fig. 3. GF's cross-section after selective etching in $\mathrm{HNO}_{3}$ : front and $45^{\circ}$ views (SEM).

\section{Chemical composition stratification in layered films}

To prevent possible errors [3] connected with comparatively small films thickness and high penetrating depth of the EDS probing $(\approx 1-2 \mu \mathrm{m})$ the X-ray microanalysis of only GF edges was realized. On the other side, another type of error is obtained in this experimental geometry: the EDS detector signal strongly depends on the film edge surface roughness. Thus, to statistically prevent the measured data scattering the programmable raster-type X-ray analysis was used.

Figure 4 demonstrates some experimental results of X-ray analysis. At first, it can be seen that distribution of Bi and Fe contents has a characteristic "gradient" near the GGG-GF interface. At second, the difference between typical single-layer and double-layer GF samples is clearly seen, too.

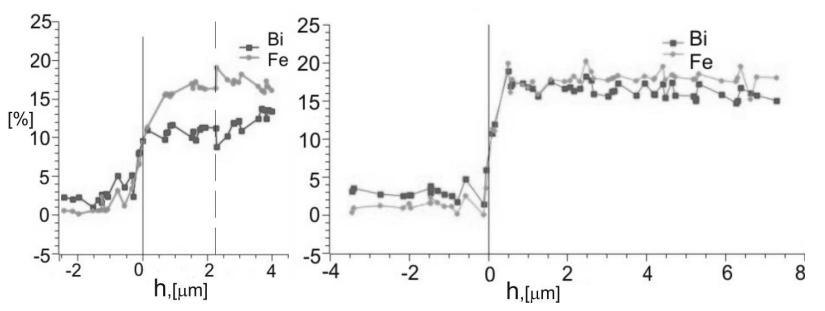

Fig. 4. Distribution of $\mathrm{Bi}$ and $\mathrm{Fe}$ in the double-layer (left) and single-layer (right) film.

It is necessary to note that typical double-layer films samples are usually characterized by two different types of DS, which can be clearly seen in polarized light (Fig. 5). As in the case of single-layer ones, they can be stable or metastable in dependence on the demagnetizing method.

Using X-ray diffractometry two different misfits were detected in these GF: between each film sublayer and substrate, correspondingly. For example, for the double-layer GF, presented in Fig. 5, $\Delta a_{1}=0.076 \AA$ and $\Delta a_{2}=0.061 \AA$. It means that the lattice mismatch between sublayers is $0.015 \AA$.

The sublayers indicate the physically different films grown on the same substrate. In our opinion, the non-uniform stress distribution and, moreover, composition stratification along the growth direction can be used to 


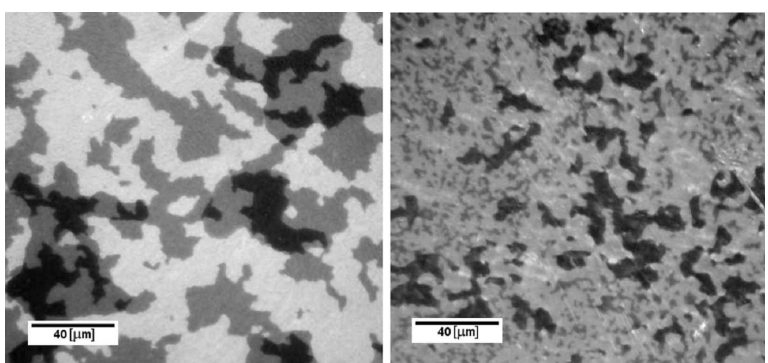

Fig. 5. Stable (left) and metastable (right) DS in double-layer GF (polarized light).

create some special types of atomic traps. But "cellular"like structure of strained GF and networks of dislocations limit the traps localization $(\approx 1-2 \mu \mathrm{m})$ : thin thermomagnetically recorded lines and dots must have zigzag binding.

\section{Potential possibilities of layered films for multilevel traps creating}

Trapping potentials for ultracold atoms are formed as the superposition of an external field and stray fields from the recorded domain patterns. Due to high $H_{\mathrm{c}}$ value the domain pattern can be stable in a certain range of changing homogeneous external field $H_{\mathrm{b}}$. The local stray field and thereby the trap "level" are practically constant. But in a case of double-layered film it is possible to operate the trap "level" in order to localize atoms on different (but fixed) distances from the garnet surface. It is a way to multilevel trap. This feature of layered GF was discussed in detail in [3].

Thermorecording of domain patterns in adjacent sublayers can be provided by technological selection of the certain values of $H_{\mathrm{c}}, 4 \pi M_{\mathrm{s}}$ and, first of all, of $T_{\mathrm{C}}$. In principle, it is necessary to provide the conditions: $H_{\mathrm{c} 1} \geq$ $H_{\mathrm{c} 2} \geq H_{\mathrm{b}}, H_{\mathrm{c} i} \approx H_{\mathrm{s} i},\left(T_{\mathrm{C} 1}-T_{\mathrm{C} 2}\right) / T_{\mathrm{C} 1} \geq 10 \%\left(H_{\mathrm{s} i}\right.$ is the saturation field of $i$ sublayer). As it is known [7], during LPE process the melt temperature determines the segregation coefficients of all garnet-forming elements. If the growth temperature decreases, the decrease of $\mathrm{Me}$ ions $(\mathrm{Ga}, \mathrm{Sm}$ or $\mathrm{Al}$ ) concentration and increase of $\mathrm{Bi}$ content in the garnet are obtained (in comparison to initial growth temperature conditions). Thus, the value and the regularity of growth temperature $T_{\mathrm{g}}$ changing at LPE were chosen in order to obtain appreciable differences in parameters, but nearly equal thicknesses of GF sublayers. In addition, it is expedient to change the velocity $\omega_{\mathrm{s}}$ of substrate (GGG) rotation. Both ways were used to provide the films composition stratification and double-layered GF samples were synthesized experimentally.

The procedure of thermorecording is described in [3] in detail and foresees two levels of laser beam power $\left(\lambda_{\mathrm{l}}=532 \mathrm{~nm}\right)$ at presence of stepped switchable homogeneous bias field. Figure 6 (left) presents non-through domain patterns thermorecorded as $2.0 \mu \mathrm{m}$ width parallel vertical lines in the top sublayer of the double-layer film; additional local switching of the bottom layer (through-type domains) is seen as a narrow portion of the image with enhanced brightness.

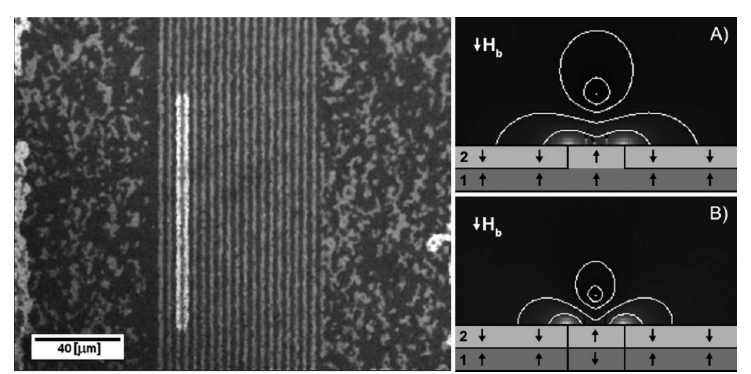

Fig. 6. Recorded domain patterns (left) and trap model (right) for double-layer GF.

Figure 6 (right) is the computer model of the corresponding atomic trap and demonstrates the main difference of the multilevel trap: it can be possible to localize "ultracold" neutral atoms at different fixed distances from GF surface using the same bias field $H_{\mathrm{b}}$. They are: $4.29 \mu \mathrm{m}(\mathrm{A}) ; 2.53 \mu \mathrm{m}$ (B) for $H_{\mathrm{b}}=80$ Oe.

\section{Conclusions}

The authors' point of view is that new experiments in the high-coercive films growth must be oriented to provide the "multilevel" atomic traps and traps having dimensions in submicron range. It is necessary to note that unlike single-layer GF the multilayer films were not tested yet experimentally as the "multilevel" atomic traps. But they can be effectively used in particular cases when manipulations with "ultracold" atoms along the vertical axis without any changes of bias field are necessary. Unfortunately, the localization degree of trapped atom clouds is limited by inevitable existence of dislocations networks in the films; the clouds geometry would be distorted maximally if their dimensions are comparable with networks segments $(1-2 \mu \mathrm{m})$.

\section{References}

[1] V. Vishnevskii, A. Nesteruk, A. Nedviga, S. Dubinko, A. Prokopov, Sens. Lett. 5, 29 (2007).

[2] A. Jaakkola, A. Shevchenko, K. Lindfors, M. Hautakorpi, E. Il'yashenko, T. Johansen, M. Kaivola, Eur. Phys. J. D 35, 81 (2005).

[3] V. Berzhansky, V. Vishnevskii, A. Nedviga, A. Nesteruk, J. Magnetics (Korea) 14, 108 (2009).

[4] V. Berzhansky, A. Nedviga, V. Vishnevskii, A. Prokopov, Solid State Phenom. 152-153, 11 (2009).

[5] D. Gualtieri, P. Tumelti, J. Appl. Phys. 57, 3879 (1985).

[6] D. Miller, R. Caruso, J. Cryst. Growth 27, 274 (1974).

[7] W. Tolksdorf, C.-P. Klages, Thin Solid Films 114, 33 (1984). 